\title{
NO CONVERGENCIA EN AMERICA LATINA
}

\author{
Carlos Dabús* \\ Fernando Delbianco \\ María Belén Zinni
}

recibido: febrero 2014 - aceptado: diciembre 2014

\begin{abstract}
Resumen
El trabajo presenta un análisis empírico sobre la convergencia para América Latina durante el periodo 1960-1998, por medio de estimaciones de datos de panel. La evidencia encontrada no indica la presencia de convergencia absoluta ni condicional, ni tampoco la existencia de clubes de convergencia. A su vez, los resultados de las estimaciones realizadas con efectos fijos muestran que no existe convergencia condicional, lo que sugiere que cada economía converge a su propio estado estacionario.
\end{abstract}

Palabras clave: crecimiento, convergencia.

Código JEL: O0-O5.

\begin{abstract}
In this paper we carry out an empirical analysis on convergence for Latin America for the 1960-1998 period, by means of panel data estimations. Our evidence does not indicate neither the presence of absolute and conditional convergence, or clubs of convergence. In turn, the fixed effects estimations results do not show conditional convergence, which suggest that each economy converges to its own steady state.
\end{abstract}

Key words: growth, convergence.

JEL Clasification: O0-O5.

* CONICET y Departamento de Economía- Universidad Nacional del Sur. E-mail: cdabus@criba.edu.ar. 


\section{INTRODUCCION}

Uno de los temas de mayor interés reciente en la literatura teórica y empírica del crecimiento ha sido la hipótesis de la convergencia. La idea de que los países pobres experimentan tasas de crecimiento más altas que los países más ricos permite predecir, en el largo plazo, un fenómeno de catching-up entre ellos. Esta predicción es postulada, en principio, por el modelo de crecimiento neoclásico, tanto en términos de convergencia absoluta o condicional. La primera se verifica cuando economías diferentes, en particular en sus parámetros, como la tasa de ahorro o de crecimiento poblacional, convergen a un mismo nivel de producto per cápita. La segunda, en cambio, se refiere a los casos en que se encuentra entre economías similares en sus parámetros y por tanto en su estado estacionario. Por tanto, aquellas economías que inicialmente se encuentran a una mayor distancia del estado estacionario experimentarán una mayor tasa de crecimiento, convergiendo con aquellas economías similares que inicialmente partieron de un nivel de producto per cápita mayor. Por el contrario, la teoría del crecimiento endógeno propone que el progreso técnico es endógeno y no predice convergencia.

En este sentido, las economías de América Latina muestran diferencias importantes en su desempeño en las últimas décadas, a la vez que constituyen un conjunto de países con un importante elemento en común: todos pertenecen a la categoría de países en vías de desarrollo.

A su vez, en términos de los aportes empíricos la mayoría de los estudios sobre convergencia se basan en regresiones de corte transversal o en el análisis de series de tiempo. Las diferencias entre ambas técnicas radican en que mientras que el primero de estos métodos no utiliza la información proveniente de la variación temporal de las variables y suponer que la muestra de economías es homogénea, estos aspectos sí son captados por los estudios de series de tiempo.

Por otra parte, el reciente resurgimiento del crecimiento de las economías latinoamericanas en la última década ha sido significativo pero a la vez heterogéneo entre las economías de la región, por lo que parece relevante determinar si este proceso estuvo asociado a la existencia de convergencia o a una mayor divergencia entre los países de la región. A su vez, los estudios empíricos no presentan un claro consenso al respecto, como se verá más adelante. Por tanto, el tema amerita la búsqueda de evidencia adicional, en particular en el caso de países en vías de desarrollo, dado que desde el seminal trabajo de Baumol (1986) para el caso de los países desarrollados hay un mayor consenso sobre convergencia. A fin de brindar tal evidencia, el presente trabajo tiene por objeto analizar la convergencia en Amé- 
rica Latina, aplicando la técnica de datos de panel con efectos fijos, los que captan diferencias no observables entre países. Se utiliza un panel de datos de 20 países latinoamericanos, con datos quinquenales para el periodo 1950-2009. Los resultados indican la ausencia de convergencia, así como el hecho de que cada economía converge a su propio estado estacionario, lo que es parcialmente compatible con las conclusiones del modelo neoclásico.

En la sección I se realiza una breve revisión de los estudios empíricos sobre convergencia para varios países, y para regiones de un mismo país. En la sección II se presentan los datos utilizados y la metodología de investigación: se definen la ecuación a estimar y las variables utilizadas. La sección III presenta los resultados de las estimaciones de convergencia para la muestra total de países latinoamericanos bajo estudio. Luego, en base a la clasificación del Banco Mundial, se divide la muestra total según los diferentes grupos de ingreso -sobre la base del nivel de ingreso inicial- y se estima la convergencia en cada uno de ellos. Posteriormente se realizan estimaciones que contemplan la presencia de factores específicos (efectos fijos). Los resultados indican "no convergencia" en la región, siendo que cada país converge a su propio estado estacionario. Finalmente, en la sección IV se presen$\tan$ las conclusiones.

\section{EVIDENCIA EMPIRICA}

La literatura empírica sobre convergencia se ha basado fundamentalmente en la estimación de regresiones múltiples para una amplia muestra de países (desarrollados y en desarrollo) durante un cierto período de tiempo. Estos estudios intentan identificar los factores que explican las diferencias en los procesos de crecimiento entre países. En esta corriente se destacan los trabajos empíricos "à la Barro", cuya metodología consiste en estimar la tasa de crecimiento del producto per cápita en función de un amplio conjunto de variables. La variable dependiente es la tasa promedio de crecimiento a lo largo de un período de tiempo. Algunas variables explicativas están expresadas en valores promedio para todo el período de estudio (por ejemplo la relación inversión/producto, la tasa de crecimiento poblacional, la tasa promedio del gasto público, etc.) y otras valuadas al inicio del período (índices de educación, PBI per cápita inicial).

La ausencia de convergencia de los niveles de ingreso per cápita en una amplia muestra de países ha sido verificada por numerosos trabajos empíricos (por ejemplo De Long (1988) y Barro (1991), entre otros). A partir de dicha evidencia, Barro (1991), Mankiw, Romer y Weil (1992), y Barro y Sala-i-Martin (1992) res- 
tablecieron la relación existente entre la hipótesis de la convergencia y el modelo neoclásico. En estos trabajos se observa que la tasa de crecimiento de economías similares, en términos de los parámetros que determinan el estado estacionario, como la tasa de ahorro y de crecimiento poblacional, está positivamente correlacionada con la distancia que la separa de su propio estado estacionario, hecho que es conocido en la literatura como $\beta$ convergencia condicional o relativa. Asimismo, es menos frecuente la evidencia de $\sigma$ convergencia, la que, a diferencia de la anterior, se verifica cuando la dispersión del producto real per cápita entre países tiende a reducirse en el tiempo.

Empíricamente se han presentado dos formas de "condicionar" las estimaciones. La primera es limitar el estudio a conjuntos de economías "similares", con lo cual tenderían al mismo estado estacionario. La literatura que utiliza esta metodología ha analizado, por ejemplo, los países de la OECD, los estados que forman los Estados Unidos, las prefecturas japonesas, las Comunidades Autónomas de España, los cantones suizos y distintas regiones de países europeos (Barro y Sala-i-Martin, 1992 y Sala-i-Martin, 1996). La segunda manera de condicionar los datos es mediante la estimación de regresiones múltiples. La idea es introducir variables adicionales que aproximen al estado estacionario.

Por otro lado, los estudios empíricos han evaluado la convergencia tanto entre los países desarrollados y en desarrollo (ver por ejemplo Rodrik,2011), como simplemente en alguno de estos grupos (por ejemplo Baumol, 1986), que analiza la convergencia en un conjunto de países desarrollados), así como para regiones de un mismo país, como es el trabajo de Duncan y Fuentes, 2006 para las regiones de Chile. En líneas generales la evidencia se puede agrupar en tres tipos de investigaciones: los estudios a nivel internacional, incluyendo países con diferente nivel de desarrollo, análisis de convergencia regional, es decir grupos de países de ciertas regiones del mundo (por ejemplo la Unión Europea o América Latina), y finalmente estudios para regiones de un país. La literatura muestra como resultados más frecuentes divergencia absoluta y convergencia condicional.

El cuadro 1 resume algunos de los estudios de convergencia. El cuadro 1.1 muestra que a nivel internacional se ha encontrado evidencia muy variada, predominando la existencia de divergencia absoluta, convergencia condicional para países de áreas económicas comunes y convergencia heterogénea o clubes de convergencia. Similarmente, Azomahou et al. (2011), Cavenaile and Dubois (2011) y Crespo-Cuaresma et al. (2008) encuentran convergencia entre estados de la Unión Europea. Estos últimos casos constituyen una especie de "híbrido", dado que no son trabajos de convergencia regional, pero sí a nivel de regiones 
económicas que engloban a varios países. Además, con respecto a la velocidad de convergencia, Azomahou et. al. (2011) encuentran que la velocidad es mayor en los países emergentes. Incluso si se pasa de un estudio de corte transversal a uno de panel, la velocidad estimada es aún mayor (toma solo unas décadas para estos países recorrer la mitad de camino hacia su estado estacionario estimado).

A su vez, para el caso de América Latina el cuadro 1.2 indica que la evidencia no es concluyente, aunque sí un predominio de convergencia condicional y divergencia global. Sin embargo, no se encuentra convergencia absoluta en la región. Por ejemplo, Madariaga et al. (2003), sobre la base de regresiones de corte transversal, encuentran evidencia de $\beta$ convergencia condicional en el Mercosur (1985-2000), así como un incremento en la dispersión del ingreso per cápita en ambos bloques económicos y una desaceleración de la $\beta$ convergencia en las unidades del Mercosur a partir del año 1991.

Cuadro 1.1: Estudios Empíricos a nivel internacional para países con diferente nivel de desarrollo

\begin{tabular}{|c|c|c|c|}
\hline $\begin{array}{l}\text { Estudios Empíricos } \\
\text { a nivel internacional } \\
\text { para economías con } \\
\text { diferente grado de } \\
\text { desarrollo }\end{array}$ & Método & Muestra & Resultado \\
\hline $\begin{array}{l}\text { Crespo-Cuaresma, } \\
\text { Ritzberger-Grüwald } \\
\text { y Silgoner (2008) }\end{array}$ & Datos de Panel & $\begin{array}{l}\text { Países de la Unión } \\
\text { Europea }\end{array}$ & $\begin{array}{l}\text { Convergencia entre } \\
\text { estados de la Unión } \\
\text { Europea posterior } \\
\text { a la vigencia del } \\
\text { acuerdo de Unión } \\
\text { Económica }\end{array}$ \\
\hline $\begin{array}{l}\text { Artelaris, Arvani- } \\
\text { tidis y Petrakos } \\
(2011)\end{array}$ & $\begin{array}{l}\text { Mínimos cuadrados } \\
\text { ponderados polinó- } \\
\text { micos (cuadráticos) }\end{array}$ & $\begin{array}{l}\text { Muestra } \\
\text { internacional de } \\
\text { países con diferente } \\
\text { nivel de ingreso }\end{array}$ & $\begin{array}{l}\text { Convergencia entre } \\
\text { países de ingreso } \\
\text { bajo y medio bajo, } \\
\text { y divergencia al } \\
\text { incluir países de } \\
\text { ingreso medio alto } \\
\text { y alto }\end{array}$ \\
\hline $\begin{array}{l}\text { Azomahou, El } \\
\text { guardighi, Nguyen- } \\
\text { Van y Pham (2011) }\end{array}$ & $\begin{array}{l}\text { Estimaciones } \\
\text { de un modelo } \\
\text { parcialmente } \\
\text { Semiparamétrico }\end{array}$ & $\begin{array}{l}157 \text { regiones de } 9 \\
\text { países de la Unión } \\
\text { Europea }\end{array}$ & $\begin{array}{l}\text { Convergencia } \\
\text { heterogénea } \\
\text { dependiendo del } \\
\text { nivel de ingreso de } \\
\text { los países }\end{array}$ \\
\hline
\end{tabular}




\begin{tabular}{|c|c|c|c|}
\hline $\begin{array}{l}\text { Cavenaile and } \\
\text { Dubois (2011) }\end{array}$ & Datos de Panel & $\begin{array}{l}\text { Países miembros de } \\
\text { la Unión Europea } \\
\text { con diferente grado } \\
\text { de desarrollo }\end{array}$ & $\begin{array}{l}\text { Encuentran } \\
\text { grupos o clubes de } \\
\text { convergencia entre } \\
\text { los nuevos y viejos } \\
\text { miembros de la UE }\end{array}$ \\
\hline $\mathrm{Hu}(2011)$ & $\begin{array}{l}\text { Cross Section y } \\
\text { Datos de Panel }\end{array}$ & $\begin{array}{l}157 \text { países no } \\
\text { petroleros, } 28 \text { países } \\
\text { desarrollados y } 23 \\
\text { emergentes }\end{array}$ & $\begin{array}{l}\text { Convergencia } \\
\text { condicional }\end{array}$ \\
\hline Rodrick (2011) & $\begin{array}{l}\text { Test tradicionales de } \\
\text { convergencia }\end{array}$ & $\begin{array}{l}\text { Muestra } \\
\text { internacional } \\
\text { de varios países } \\
\text { desarrollados y en } \\
\text { desarrollo }\end{array}$ & $\begin{array}{l}\text { Convergencia } \\
\text { absoluta a } \\
\text { nivel sectorial } \\
\text { (manufacturas } \\
\text { y servicios } \\
\text { sofisticados) } \\
\end{array}$ \\
\hline $\begin{array}{l}\text { Cuestas, Monfort } \\
\text { y Ordóñez (2012) }\end{array}$ & $\begin{array}{l}\text { Análisis por medio } \\
\text { de Clusters }\end{array}$ & $\begin{array}{l}14 \text { países miembros } \\
\text { de la Unión } \\
\text { Europea }\end{array}$ & $\begin{array}{l}\text { Convergencia } \\
\text { heterogénea, con la } \\
\text { formación de clubes } \\
\text { de convergencia } \\
\text { dentro de la UE }\end{array}$ \\
\hline $\begin{array}{l}\text { Rabanal, Cristian } \\
(2012)\end{array}$ & $\begin{array}{l}\text { Estimaciones á la } \\
\text { Barro, Econometría } \\
\text { espacial y Matriz de } \\
\text { Quah }\end{array}$ & $\begin{array}{l}\text { Muestra } \\
\text { internacional de } 101 \\
\text { países }\end{array}$ & $\begin{array}{l}\text { Convergencia en } \\
\text { los países de mayor } \\
\text { ingreso con el } \\
\text { método à la Barro, } \\
\text { y convergencia } \\
\text { generalizada con el } \\
\text { de Quah }\end{array}$ \\
\hline $\begin{array}{l}\text { Sperlich, y Sperlich } \\
\text { (2012) }\end{array}$ & $\begin{array}{l}\text { Datos de Panel } \\
\text { con efectos fijos } \\
\text { (GLS-Regression } \\
\text { with panel- } \\
\text { specific AR(1), } \\
\text { heteroscedasticity } \\
\text { and fixed effects). }\end{array}$ & $\begin{array}{l}\text { Varios países } \\
\text { pertenecientes } \\
\text { a tres áreas de } \\
\text { acuerdo comercial: } \\
\text { Mercosur, Sudeste } \\
\text { de Asia y África: }\end{array}$ & $\begin{array}{l}\text { Convergencia } \\
\text { impulsada por los } \\
\text { acuerdos de áreas } \\
\text { económicas en los } \\
\text { casos de Mercosur, } \\
\text { Sudeste Asiático y } \\
\text { Países Africanos. }\end{array}$ \\
\hline
\end{tabular}


Cuadro 1.2: Estudios Empíricos sobre convergencia para América Latina

Estudios
Empíricos para
América Latina

Método

Muestra

Resultado

América Latina

\begin{tabular}{llll}
\hline $\begin{array}{l}\text { Helliwell y } \\
\text { Chung (1992) }\end{array}$ & $\begin{array}{l}\text { Regresión de corte } \\
\text { transversal }\end{array}$ & $\begin{array}{l}18 \text { países } \\
\text { latinoamericanos } \\
(1960-1985)\end{array}$ & $\begin{array}{l}\beta \text { convergencia } \\
\text { condicional }\end{array}$
\end{tabular}

(1960-1985)

\begin{tabular}{llll}
\hline Rincón & Regresión con & 18 países & $\beta$ convergencia \\
Piedrahita & datos de panel con & latinoamericanos & condicional \\
$(1998)$ & efectos fijos & $(1960-1990)$ &
\end{tabular}

\begin{tabular}{|c|c|c|c|}
\hline $\begin{array}{l}\text { Cáceres } \\
\text { y Nuñez } \\
\text { Sandoval } \\
(1999)\end{array}$ & $\begin{array}{l}\text { Pruebas de raíces } \\
\text { unitarias }\end{array}$ & $\begin{array}{l}17 \text { países latinoame- } \\
\text { ricanos }\end{array}$ & $\sigma$ divergencia \\
\hline $\begin{array}{l}\text { Dobson y } \\
\text { Ramlogan } \\
(2002)\end{array}$ & $\begin{array}{l}\text { Regresión de corte } \\
\text { transversal y con } \\
\text { datos de panel y } \\
\text { efectos fijos }\end{array}$ & $\begin{array}{l}19 \text { países } \\
\text { latinoamericanos } \\
\text { (Naciones Unidas) } \\
(1960-1997)\end{array}$ & $\begin{array}{l}\beta \text { divergencia (absoluta } \\
\text { y condicional) }\end{array}$ \\
\hline $\begin{array}{l}\text { Dobson, } \\
\text { Goddard y Ra- } \\
\text { mlogan (2003) }\end{array}$ & $\begin{array}{l}\text { Pruebas de raíces } \\
\text { unitarias con datos } \\
\text { de panel }\end{array}$ & $\begin{array}{l}24 \text { países latinoame- } \\
\text { ricanos }\end{array}$ & $\begin{array}{l}\text { Convergencia parcial } \\
\text { (solamente para } \\
\text { algunos países) }\end{array}$ \\
\hline $\begin{array}{l}\text { Madariaga et } \\
\text { al. (2003) }\end{array}$ & corte transversal & Mercosur (1985-2000) & $\begin{array}{l}\beta \text { convergencia } \\
\text { condicional }\end{array}$ \\
\hline $\begin{array}{l}\text { Barrientos } \\
\text { Quiroga (2010) }\end{array}$ & $\begin{array}{l}\text { Cross Section y } \\
\text { Datos de Panel }\end{array}$ & $\begin{array}{l}\text { Muestra de } 32 \text { países } \\
\text { de Latinoamérica y el } \\
\text { Caribe }\end{array}$ & $\begin{array}{l}\text { Convergencia } \\
\text { heterogénea (más } \\
\text { de una velocidad de } \\
\text { convergencia o clubs } \\
\text { de convergencia) }\end{array}$ \\
\hline
\end{tabular}


Cuadro 1.3: Estudios Empíricos sobre convergencia regional

\begin{tabular}{|c|c|c|c|}
\hline $\begin{array}{l}\text { Estudios } \\
\text { Empíricos para } \\
\text { regiones de un } \\
\text { país }\end{array}$ & Método & Muestra & Resultado \\
\hline $\begin{array}{l}\text { Cárdenas y Pon- } \\
\text { tón (1995) }\end{array}$ & corte transversal & $\begin{array}{l}\text { Veinticuatro } \\
\text { departamentos de } \\
\text { Colombia para el } \\
\text { período 1950-1990 }\end{array}$ & $\beta$ convergencia absoluta \\
\hline Ferreira (1998) & Corte transversal & $\begin{array}{l}\text { Estados brasileños } \\
\text { para el período } \\
\text { 1939-1995 }\end{array}$ & $\begin{array}{l}\beta \text { convergencia } \\
\text { condicional }\end{array}$ \\
\hline Marina (1999) & corte transversal & $\begin{array}{l}\text { Provincias } \\
\text { argentinas entre } \\
1970 \text { y } 1995\end{array}$ & $\begin{array}{l}\beta \text { divergencia absoluta } \\
\text { y } \sigma \text { divergencia }\end{array}$ \\
\hline $\begin{array}{l}\text { Marina et al. } \\
(2000)\end{array}$ & $\begin{array}{l}\text { Corte transversal } \\
\text { incorporando } \\
\text { variables adicionales } \\
\text { de educación y } \\
\text { gasto público }\end{array}$ & $\begin{array}{l}\text { Provincias } \\
\text { argentinas entre } \\
1970 \text { y } 1995\end{array}$ & $\beta$ divergencia absoluta \\
\hline $\begin{array}{l}\text { Duncan y } \\
\text { Fuentes (2006) }\end{array}$ & $\begin{array}{l}\text { Tests de } \\
\text { convergencia } \\
\text { tradicionales y test } \\
\text { de raíces unitarias } \\
\text { en datos de panel }\end{array}$ & $\begin{array}{l}\text { Convergencia } \\
\text { regional en } \\
13 \text { regiones } \\
\text { económicas de } \\
\text { Chile }\end{array}$ & $\beta$ convergencia absoluta \\
\hline $\begin{array}{l}\text { Young, Higgins } \\
\text { y Levy (2013): }\end{array}$ & $\begin{array}{l}\text { Estimaciones por } \\
\text { medio de GMM }\end{array}$ & $\begin{array}{l}\text { Convergencia re- } \\
\text { gional dento de los } \\
\text { estados de EEUU }\end{array}$ & $\begin{array}{l}\text { Convergencia } \\
\text { Heterogénea (a } \\
\text { diferentes tasas entre } \\
\text { estados) }\end{array}$ \\
\hline $\begin{array}{l}\text { Cherodian } \\
\text { y Thirlwall } \\
(2013)\end{array}$ & $\begin{array}{l}\text { Estimaciones } \\
\text { Cross section para } \\
\beta \text { convergencia } \\
\text { condicional, } \\
\text { convergencia } \\
\text { absoluta y } \sigma \\
\text { convergencia. }\end{array}$ & $\begin{array}{l}\text { Convergencia } \\
\text { regional en } 32 \\
\text { regiones de India }\end{array}$ & Convergencia débil \\
\hline
\end{tabular}


Dentro de los estudios empíricos para regiones de un país hay indicios más favorables a la convergencia, mientras que solamente en el caso de 32 regiones de India se encuentra $\sigma$ convergencia, la que, como se mencionó anteriormente, ocurre cuando la dispersión del producto real per cápita, en este caso de estas regiones, tiende a reducirse en el tiempo. Según los resultados de Cárdenas y Pontón (1995), quienes utilizan regresiones de corte transversal, existe $\beta$ convergencia absoluta en los veinticuatro departamentos de Colombia para el período 1950-1990. Utrera y Koroch (1998) realizan estimaciones de corte transversal sobre las veinticuatro jurisdicciones de Argentina entre 1953 y 1994, encontrando evidencia de $\beta$ convergencia condicional una vez que se incluyen variables adicionales de capital humano e indicadores fiscales. Marina (1999), utilizando también regresiones de corte transversal encuentra divergencia absoluta y $\sigma$ divergencia en las provincias argentinas en distintos períodos entre 1970 y 1995. Marina et al. (2000), estudian la $\beta$ convergencia condicional incorporando las variables adicionales educación y gasto público, y encuentran que los resultados no se modifican con respecto al trabajo anterior. Ferreira (1998), utilizando regresiones de corte transversal encuentra $\beta$ convergencia condicional en los estados brasileños para el período 1939-1995. Por último, Duncan y Fuentes (2006) para Chile, Young et al., para Estados Unidos, y Cherodian y Thirwal (2013) para India) encuentran $\beta$ convergencia absoluta

Finalmente, dentro de la literatura de convergencia, se puede hacer referencia a aquellos trabajos que refinan la búsqueda de la misma, pero no a nivel internacional ni regional, sino a nivel industria. Entre estos trabajos podemos hallar a Rodrick (2011) y Barrientos Quiroga (2010), que toman en cuenta los procesos de industrialización a la hora de hablar de convergencia. Los resultados resaltan el hecho de que existe cierta convergencia (y a mayor velocidad) entre aquellos países/regiones que tuvieron procesos de industrialización exitosos.

En resumen, la literatura empírica sobre convergencia presenta resultados claramente disímiles. En particular, para el caso de América latina se observa un cierto predominio de divergencia global y convergencia condicional. En cambio, los estudios realizados en el ámbito nacional encuentran evidencia a favor de la existencia convergencia entre estados de un mismo país.

\section{METODOLOGIA Y DATOS UTILIZADOS}

La metodología habitualmente utilizada en los estudios empíricos de la convergencia ha seguido dos líneas de investigación principales: "el análisis de regresión" y "el análisis de la dinámica de la distribución". La primera comprende las técnicas de datos de panel y la de estudios de corte transversal, mientras que la 
segunda se basa en examinar directamente como cambia la distribución del ingreso entre países per cápita de distintas economías a lo largo del tiempo. En este sentido, Quah (1993), demuestra cómo la existencia de $\beta$ convergencia es compatible con distribuciones de ingresos per cápita que tienden a permanecer constantes o incluso con aquellas cuya amplitud global tienda a aumentar en el tiempo. Este enfoque surge como alternativa al análisis de regresión para estimar la convergencia, porque considera aspectos relacionados con la movilidad, la estratificación y la polarización de la distribución del ingreso entre economías. De esta manera, presenta un esquema que él mismo denomina "la dinámica de la distribución", cuya técnica más simple es la creación de matrices de movilidad (o de transición) sobre las cuales se analizan las posiciones relativas de las economías en el tiempo.

Dentro de cada uno de estos enfoques puede encontrarse una gran cantidad de trabajos empíricos sobre convergencia. Sin embargo, la literatura no presenta evidencia suficiente respecto a estudios para muestras de economías relativamente homogéneas en lo que se refiere a sus niveles de desarrollo y al hecho de compartir un área geográfica común (Helliwell y Chung, 1992; Cellini, 1997). Además, son escasos los estudios para regiones de países que utilizan la técnica de datos de panel (y dentro de estos solo en dos casos se encuentra convergencia condicional para América Latina, mostrada en la evidencia del cuadro 1.3). Como se explicó anteriormente, esta técnica presenta claras ventajas sobre las de corte transversal, por lo que amerita cubrir este aspecto no estudiado en forma exhaustiva en la literatura. Por tal motivo, se analiza la convergencia para países de América Latina en el marco del análisis de regresión utilizando datos de panel.

Por otra parte, en la literatura del crecimiento pueden encontrarse distintas especificaciones ad hoc en lo que respecta a la elección de las variables utilizadas para controlar el estado estacionario. En las estimaciones del presente trabajo se incluyen como variables explicativas aquellas encontradas como robustas por Levine y Renelt (1992), es decir el PBI per cápita inicial, la relación inversión/ producto, el capital humano y el crecimiento poblacional. Por tanto en el trabajo se considera la siguiente ecuación de $\beta$ convergencia condicional:

$$
\gamma \mathrm{i}, \mathrm{t}=\alpha+\beta \log \left(y_{0}\right)+\varphi_{1} X_{1}+\varphi_{2} X_{2}+\varphi_{3} X_{3}+\varphi_{4} X_{4}+\varepsilon_{i . t}
$$

\section{Donde}

$\gamma_{\mathrm{i}, \mathrm{t}}$ : Tasa de crecimiento del PBI per cápita (en porcentaje anual)

$\mathrm{y}_{0}$ : PBI per cápita inicial 1950 (en dólares constantes 2000)

$\mathrm{X}_{1}$ : Inversión doméstica bruta (porcentaje del PBI)

$\mathrm{X}_{2:}$ Tasa de crecimiento poblacional (en porcentaje anual)

$\mathrm{X}_{3}$ : Capital humano (años promedio de educación de población mayor de 25 años) 
$\mathrm{X}_{4}$ : Coeficiente de Apertura $(\mathrm{X}+\mathrm{M} / \mathrm{PBI})$

$\mathrm{T}$, t: periodo final e inicial, respectivamente.

Siguiendo a Barro (1997), las variables nivel inicial del producto real per cápita y capital humano son denominadas variables de estado, ya que las mismas representan el estado inicial de la economía. Por tal motivo, son medidas al momento inicial del período. El resto de las variables explicativas, en cambio, dependen de las decisiones de los agentes, por lo que se denominan variables de elección o de contexto. Estas últimas son medidas como valores promedio del período. A su vez, se utiliza como variable proxy de capital humano a los años promedio de educación de la población mayor de 25 años. Esta medida es un indicador más preciso para representar al capital humano disponible para la producción, si se lo compara con las medidas tradicionalmente incluidas como variables "proxy" del nivel de educación, es decir las tasas de escolarización (Barro-Lee, 1993), dado que esta última solo tendría impacto productivo en el futuro. Asimismo, los resultados obtenidos no cambian sustancialmente al utilizar las medidas consideradas usualmente como alternativas a ésta, como por ejemplo el porcentaje de población mayor de 25 años con educación primaria, secundaria y superior completa.

Los datos utilizados han sido obtenidos con una frecuencia de intervalos de cinco años, de la siguiente manera: la tasa de crecimiento del producto per cápita, el producto per cápita, la participación de la inversión en el producto, las exportaciones, importaciones, el PBI y la tasa de crecimiento poblacional son extraídos de la base de datos de la CEPAL, mientras que la variable de capital humano es extraída de la base de datos de Barro-Lee (2010) ${ }^{1}$.

\section{II.1 Datos de Panel}

Además de las diferencias relacionadas a las capacidades económicas y tecnológicas entre países, existen también las referidas a ciertos aspectos culturales e institucionales de una sociedad. Estas diferencias no logran ser captadas por una regresión de corte transversal, por lo que la incidencia de dichos factores queda en el residuo de la regresión, creando un sesgo por omisión de variables. Es decir, si una o más variables explicativas están correlacionadas con la variable omitida, se incurre en error de sesgo de la estimación de los parámetros. Por otra parte, las estimaciones de corte transversal utilizan las variables medidas en promedio a lo largo del período, con lo cual se pierde la información proveniente

1 Barro, Robert and Jong-Wha Lee, April 2010, "A New Data Set of Educational Attainment in the World, 1950-2010." NBER Working Paper No. 15902. 
de la evolución temporal de las variables, mientras que las estimaciones de datos de panel captan estas diferencias, por lo que son una mejor aproximación para superar estos problemas.

Por otro lado, una dificultad de estas técnicas es la periodicidad de los datos. La consideración de períodos excesivamente cortos, introduce perturbaciones cíclicas en el análisis que oscurecen la dinámica de largo plazo. Por otra parte, algunas de las variables de interés no se encuentran disponibles con tal frecuencia, como es el caso de las variables de capital humano. Por estas razones, la literatura considera generalmente lapsos temporales de entre cinco y diez años (Barro y Sala-i-Martin (1992), Islam (1995), Caselli, Esquivel y Lefort (1996)), aunque aplicaciones con intervalos más pequeños también son frecuentes (Lee, Pesaran y Smith,1997, 1998; De la Fuente, 1998).

Por tanto, en base a la disponibilidad de datos, en el presente estudio se trabaja con promedios quinquenales para una muestra de 19 países latinoamericanos durante el período 1950-2009². Las variables de estado (producto per cápita y capital humano) se consideran al inicio de cada uno de ellos, por lo que para cada país se dispone de ocho observaciones variables (ocho períodos). De este modo, cuando t+T es 1965, t es 1960 y los valores de las variables de elección o de contexto son el promedio de sus valores entre 1960 y 1964.

\section{EVIDENCIA EMPIRICA: NO CONVERGENCIA EN LATINOAMÉRICA}

En esta sección se presentan los resultados de las estimaciones de convergencia para la muestra total de países y para los distintos grupos antes definidos. En primer lugar, el gráfico 1 sugiere la existencia de divergencia absoluta en la región: en general los países con mayor ingreso per cápita han logrado un mayor crecimiento económico promedio a lo largo del periodo en estudio.

2 Dado que la frecuencia de los datos de la variable capital humano (5 años) en la base de Barro Lee (2000), siguiendo a Islam (1995), Rincon Piedrahita (1998) y Dobson y Ramlogan (2002) se utilizan valores promedios cada cinco años. Los valores promedio abarcan los períodos 1950-1954, 1955-1959, 1960-1964, 1965-1969, 1970-1974, 1975-1979, 1980-1984, 1985-1989, 1990-1994, 1995-1999, 2000-2004, 2005-2009. A su vez se toma como último año de la muestra el 2009 debido al hecho de que los datos son quinquenales, por lo que si se actualizarán los datos aun no se podría obtener un quinquenio completo. 
El gráfico 1 muestra una línea de la tendencia con pendiente positiva entre el PBI per cápita inicial y el crecimiento promedio de los países de la región, lo que indicaría la existencia de divergencia entre los mismos, es decir un progresivo alejamiento de los países de mayor ingreso de los más pobres. A su vez, este resultado no cambia si se excluye a Haití de la muestra, por ser este un caso particular, dado que es el único país de ingreso bajo, hecho que se tendrá nuevamente en cuenta en las estimaciones presentadas más abajo.

Por otro lado, en los cuadros 2 y 3 se agrupan los países según su nivel de ingreso per cápita, los que muestran que los países de ingreso medio alto crecieron a una tasa superior $(1,85 \%)$ a la de los otros dos grupos $(1.15 \%$ para los países de ingreso medio bajo y $1,14 \%$ para el caso de Haití), lo que indicaría que los grupos de países agrupados en sus respectivos niveles de grupos de ingreso no convergen entre sí.

Gráfico 1: Media de crecimiento del producto per capita (1950-2009) versus logaritmo del ingreso per cápita en 1950.

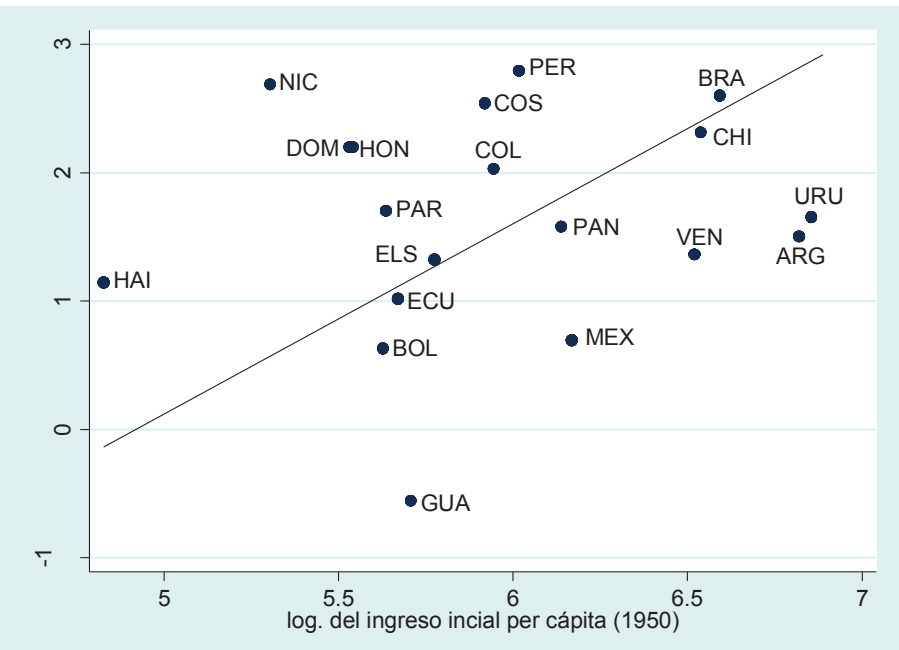

Fuente: elaboración propia en base a datos del Banco Mundial. 
Las abreviaturas correspondientes a cada país son especificadas en el siguiente cuadro anexo del gráfico anterior.

\begin{tabular}{|c|c|}
\hline País & Abreviatura \\
\hline Argentina & $\arg$ \\
\hline Bolivia & bol \\
\hline Brasil & bra \\
\hline Chile & chi \\
\hline Colombia & col \\
\hline Costa Rica & $\cos$ \\
\hline Ecuador & ecu \\
\hline El Salvador & els \\
\hline Guatemala & gua \\
\hline Haití & hai \\
\hline Honduras & hon \\
\hline México & $\operatorname{mex}$ \\
\hline Nicaragua & nic \\
\hline Panamá & pan \\
\hline Paraguay & par \\
\hline Perú & per \\
\hline República Dominicana & dom \\
\hline Uruguay & uru \\
\hline Venezuela & ven \\
\hline
\end{tabular}

En segundo lugar, los países que superaban el promedio del producto per cápita en 1960 crecieron a una tasa superior a la de los países con un producto per cápita inferior (ver cuadro 3). Por otra parte, si se compara la tasa de crecimiento promedio de los países de ingreso medio -alto y bajo en forma conjunta- con la de los países de ingreso bajo se obtiene una conclusión similar ${ }^{3}$. En síntesis, tanto el gráfico como los cuadros anteriores sugieren divergencia en el proceso de crecimiento de la región.

3 Los resultados de la aplicación del test de diferencia de medias en cada caso muestran que sólo las medias del grupo de ingreso medio bajo y de ingreso bajo son significativamente diferentes al 10\%. 
Cuadro 2. Tasa de crecimiento anual promedio por grupos de países 1950-2009 Clasificación según el Criterio del Banco Mundial $(*)(* *)$

\begin{tabular}{llll}
\hline $\begin{array}{l}\text { Países de Ingreso } \\
\text { Medio Alto (MA) }\end{array}$ & $\begin{array}{l}\text { Países de Ingreso } \\
\text { Medio Bajo (MB) }\end{array}$ & $\begin{array}{l}\text { Países de Ingreso } \\
\text { Medio Alto y Medio } \\
\text { Bajo (MA y MB) }\end{array}$ & $\begin{array}{l}\text { Países de Ingreso } \\
\text { Medio Bajo y Bajo } \\
\text { (MB y B) }\end{array}$ \\
\hline $1,85 \%$ & $1,15 \%$ & $1,65 \%$ & $1,14 \%$ \\
\hline
\end{tabular}

(*) La clasificación del Banco Mundial considerando el PBI per cápita: Las economías se dividen de acuerdo con el INB per cápita de 2008, el que se calcula usando el método Atlas del Banco Mundial. Los grupos son: ingresos bajos, US\$975 o menos; ingresos medianos bajos, US\$976 a US\$3.855; ingresos medianos altos, US\$3.856 a US\$11.905; e ingresos altos por encima de ese valor.

(**) En la clasificación de las columnas 3 y 4 se realizan las siguientes agrupaciones. En la tercera columna se calcula el promedio de los países medio bajo y medio alto en conjunto. En la cuarta columna se incluye a Haití, único país de ingreso bajo en la muestra, en el cálculo del crecimiento promedio junto a los países de ingreso medio-bajo.

Cuadro 3. Tasa de crecimiento anual promedio por grupos de países 1950-2009. Criterio del producto per cápita promedio en 1950.

Países que superan el producto per cápita promedio de 1950
Países por debajo del producto per cápita promedio de 1950

A fin de determinar si los resultados anteriores mantienen a continuación se estudia el problema por medio del análisis de regresión. En primer lugar se estima la $\beta$ convergencia absoluta en el conjunto de países latinoamericanos. En tal sentido, las regresiones de la tabla 1 se realizan con el método Mínimos Cuadrados Ordinarios (OLS) robustos, es decir corregidos por heterocedasticidad por medio de la corrección de White.

Los resultados presentados en la primera columna corresponden a la estimación de la muestra completa de países basada en la ecuación (1), sin incluir las variables que se utilizan para controlar el estado estacionario. Estos indican la ausencia de convergencia absoluta, dado que se encuentra un coeficiente $\beta$ no significativo. La columna 2 muestra los resultados de las estimaciones sobre convergencia condicional, realizadas en base a la misma ecuación. En este caso no se consideran efectos fijos y se incluyen las variables de control sugeridas por Levine y Renelt (1992), que son las cuatro variables de control del estado es- 
tacionario antes mencionadas. Además se supone la homogeneidad de todos los parámetros entre países, es decir, considera un coeficiente para cada variable que es común para todas las unidades de corte transversal. Los resultados muestran un coeficiente positivo y significativo del logaritmo natural del producto per cápita indicaría la presencia de $\beta$ divergencia condicional. A su vez, en la tercera columna se presentan los resultados de las estimaciones excluyendo a Haití; nuevamente se observa que los resultados no cambian al excluir a Haití de la muestra, por lo que son robustos a la inclusión o exclusión de dicho país.

Tabla 1.1. Estimación de convergencia para América Latina por MCO (*)

\begin{tabular}{llll}
\hline Variable & $\begin{array}{l}\text { Muestra Completa } \\
\text { (i) }\end{array}$ & $\begin{array}{l}\text { Muestra Comple- } \\
\text { ta (ii) }\end{array}$ & $\begin{array}{l}\text { Muestra Completa } \\
\text { (iii) } \\
(\text { sin Haití) }\end{array}$ \\
\hline Inversión & 0.2034 & 0.2058 \\
& $(0.000)$ & $(0.000)$ \\
\hline Crec. Poblacional & & 0.2868 & 0.2748 \\
& & $(0.239)$ & $(0.273)$ \\
\hline Coef. de Apertura & & 0.4723 & 0.9745 \\
\hline Capital Humano & & $(0.039)$ & $(0.07)$ \\
\hline PBI per cápita Inicial & 0.2073 & 0.0542 & 0.0344 \\
& $(0.512)$ & $(0.453)$ & $(0.664)$ \\
\hline & 0.4189 & $(0.085)$ & 0.0017 \\
& $(0.825)$ & $(0.005)$ & $(0.011)$ \\
\hline
\end{tabular}

(*) Los valores entre paréntesis indican el p-value asociado al estadístico "t" 
Tabla 1.2: Estimación de la Ecuación de Crecimiento por MCO robustos (*)

\begin{tabular}{|c|c|c|c|}
\hline Variable & $\begin{array}{l}\text { Medio - Alto } \\
\text { (i) }\end{array}$ & $\begin{array}{l}\text { Medio - Bajo } \\
\text { (ii) }\end{array}$ & $\begin{array}{l}\text { Medio-Bajo } \\
\text { (iii) } \\
\text { (sin Haití) }\end{array}$ \\
\hline \multirow[t]{2}{*}{ Inversión } & 0.2207 & 0.1763 & 0.1801 \\
\hline & $(0.000)$ & $(0.000)$ & $(0.000)$ \\
\hline \multirow[t]{2}{*}{ Crec. Poblacional } & -0.3477 & 1.2648 & 1.4738 \\
\hline & $(0.201)$ & $(0.080)$ & $(0.062)$ \\
\hline \multirow[t]{2}{*}{ Coef. de Apertura } & 1.1701 & 2.0222 & 1.0499 \\
\hline & $(0.083)$ & $(0.045)$ & $(0.339)$ \\
\hline \multirow[t]{2}{*}{ Capital Humano } & -0.1951 & 0.1726 & 0.1909 \\
\hline & $(0.105)$ & $(0.090)$ & $(0.097)$ \\
\hline \multirow[t]{2}{*}{$\begin{array}{l}\text { PBI per cápita } \\
\text { inicial }\end{array}$} & 0.0008 & 0.0046 & -0.0054 \\
\hline & $(0.266)$ & $(0.293)$ & $(0.399)$ \\
\hline \multirow[t]{2}{*}{ Constante } & 1.5641 & -5.916 & -3.2301 \\
\hline & $(0.237)$ & $(0.038)$ & $(0.321)$ \\
\hline
\end{tabular}

(*) Los valores entre paréntesis indican el p-value asociado al estadístico "t"

Por otro lado, dado que la hipótesis de convergencia condicional implica suponer que los países con características estructurales parecidas convergen entre sí independientemente de sus condiciones iniciales, Galor (1996) presenta una tercer definición, más restrictiva que las anteriores, la hipótesis de los clubes de convergencia, según la cual, los países con idénticos estados estacionarios convergen entre sí siempre que sus condiciones iniciales sean similares. Esta hipótesis ha sido estudiada generalmente dentro del análisis de la dinámica de la distribución. Sin embargo, es posible introducirla en el análisis de regresión siguiendo las predicciones de los modelos de crecimiento con trampas de pobreza, que dan lugar a 
la existencia de múltiples estados estacionarios estables. Estos suponen una función de producción que presenta rendimientos decrecientes para bajos niveles de capital y rendimientos crecientes para niveles más altos. Esta forma de la función agregada de producción puede deberse a que las economías con bajos niveles de desarrollo tienden a dedicarse principalmente a la agricultura, un sector donde prevalecen los rendimientos decrecientes del capital. A medida que la economía se desarrolla, la actividad económica se concentra en la industria y los servicios, los que pueden presentar rendimientos crecientes de los factores debido a que la economía explota los beneficios del learning-by-doing y la división del trabajo. Cuando estos beneficios se ven agotados, la economía presenta nuevamente rendimientos decrecientes en los factores de producción. Esto da lugar a entonces la existencia de dos estados estacionarios estables, y entre medio de ellos uno inestable. La trampa de pobreza se presenta para bajos niveles de capital, dado que el intento de los países de salir del estado estacionario "bajo" se ve frustrado por una tendencia a retornar a él.

Las tres primeras columnas de la Tabla 1.2 muestran los resultados de las estimaciones incluyendo los grupos de países señalados en la regresión. La diferencia que existe entre las columnas 2 y 3 es que en último caso se excluye a Haití de la muestra. Esta exclusión se debe a que este país es el único que se encuentra en la categoría de país de ingreso bajo de toda la muestra. Además, los resultados indican que solo en algunos casos las variables de control son significativas y con el signo esperado. Para la variable de inversión, se puede notar que los resultados son iguales para todas las especificaciones utilizadas, siendo en todos los casos significativa y con un coeficiente de alrededor de 0.20 . Luego, las variables de capital humano, coeficiente de apertura y crecimiento poblacional son solo significativas en alguna de las especificaciones realizadas, pero en general solamente al $10 \%$.

Asimismo, cuando se divide la muestra por grupo de países, el logaritmo natural del producto per cápita inicial no es significativo cuando se toman estimaciones robustas. En este sentido es importante notar que todos los análisis anteriores, realizados sobre la base de la ecuación (1), suponen que las economías bajo estudio son relativamente homogéneas. Es decir, sólo tienen en cuenta las diferencias que captan las variables explícitamente incorporadas para controlar el estado estacionario. Sin embargo, al considerar un conjunto de países, es probable que existan diferencias no observables (o de difícil medición, más aún en países en desarrollo), relacionadas, con aspectos sociales e institucionales que influyen sobre el estado estacionario de la economía. 
A fin de captar tales factores se introducen efectos fijos en la estimación de la convergencia. Las tablas 2.1 y 2.2 muestran los resultados de las estimaciones con efectos fijos. En estas se observa que, al igual que en las estimaciones con MCO robustos, los resultados obtenidos con datos de panel también indican que las economías latinoamericanas no convergen hacia un estado estacionario común; de hecho, el signo positivo del parámetro asociado al PBI per cápita inicial indica que no existe $\beta$ convergencia absoluta. Más aún, en el caso en que se toma la muestra global sin diferenciarse por grupo de países, dicha variable tiene signo positivo y es significativa, lo que estaría indicando la existencia de divergencia.

Tabla 2.1. Estimación de panel (*).

\begin{tabular}{|c|c|c|c|}
\hline Variable & $\begin{array}{l}\text { Muestra Completa } \\
\text { (i) }\end{array}$ & $\begin{array}{l}\text { Muestra Completa } \\
\text { (ii) }\end{array}$ & $\begin{array}{l}\text { Muestra Completa } \\
\text { (iii) } \\
\text { (sin Haití) }\end{array}$ \\
\hline \multirow[t]{2}{*}{ Inversión } & & 0.2059 & 0.2086 \\
\hline & & $(0.000)$ & $(0.000)$ \\
\hline \multirow[t]{2}{*}{ Crec. Poblacional } & & 0.3951 & 0.3937 \\
\hline & & $(0.162)$ & $(0.179)$ \\
\hline \multirow[t]{2}{*}{ Coef. de Apertura } & & 1.6857 & 1.6180 \\
\hline & & $(0.010)$ & $(0.024)$ \\
\hline \multirow[t]{2}{*}{ Capital Humano } & & 0.0261 & 0.0202 \\
\hline & & $(0.791)$ & $(0.847)$ \\
\hline \multirow[t]{2}{*}{$\begin{array}{l}\text { PBI per cápita } \\
\text { inicial }\end{array}$} & 0.0004 & 0.0024 & 0.0022 \\
\hline & $(0.604)$ & $(0.024)$ & $(0.054)$ \\
\hline \multirow[t]{2}{*}{ Constante } & 1.4584 & -2.4408 & -2.2574 \\
\hline & $(0.001)$ & $(0.042)$ & $(0.076)$ \\
\hline
\end{tabular}

(*) Los valores entre paréntesis indican el p-value asociado al estadístico "t" 
Tabla 2.2. Estimación de panel por grupos (*).

\begin{tabular}{lll}
\hline Variable & $\begin{array}{l}\text { Medio - Alto } \\
(\mathrm{i})\end{array}$ & $\begin{array}{l}\text { Medio -Bajo } \\
\text { (ii) }\end{array}$ \\
\hline Inversión & 0.2229 & 0.1761 \\
& $(0.000)$ & $(0.000)$ \\
\hline Crec. Poblacional & -0.2313 & 1.3059 \\
& $(0.552)$ & $(0.025)$ \\
\hline Coef. de Apertura & 2.4040 & 2.0601 \\
Capital Humano & $(0.014)$ & $(0.013)$ \\
& -0.1867 & 0.1657 \\
& $(0.244)$ & $(0.166)$ \\
\hline \multirow{2}{*}{ PBI per cápita inicial } & 0.9039 & 1.0763 \\
& $(0.182)$ & $(0.248)$ \\
\hline \multirow{2}{*}{ Constante } & -4.4738 & -10.7381 \\
\hline
\end{tabular}

(*) Los valores entre paréntesis indican el p-value asociado al estadístico " $t$ "

Tampoco se encuentra evidencia de $\beta$ convergencia condicional dentro de los grupos de países que poseen niveles de desarrollo inicial similares (no se verifica la hipótesis de clubes de convergencia). Sobre la base de estos resultados se concluyen que la tasa de crecimiento de los países latinoamericanos es afectada por factores específicos de cada país. Esta hipótesis se verifica utilizando la técnica de datos de panel con efectos fijos. En consecuencia, la información contenida en los efectos fijos es lo que determina el estado estacionario al que converge cada economía en particular.

Por último, la evidencia de "no convergencia" obtenida en este trabajo para América Latina constituye un resultado que sugiere la existencia de factores subyacentes a cada economía en particular que las impulsa a no tender hacia un estado estacionario común, tanto a nivel global como para países de ingreso similar. Una posible línea de trabajo futuro es explorar cuales son tales factores, y en qué medida pueden potenciar un alejamiento o acercamiento futuro del nivel de ingreso de los países de la región. 


\section{CONCLUSIONES}

Los trabajos sobre convergencia para Latinoamérica en general estudian existencia de $\beta$ convergencia (absoluta y condicional) sobre la base de regresiones de corte transversal. Estos análisis no permiten captar la heterogeneidad no observable entre países, es decir, las diferencias sociales, institucionales y tecnológicas existentes entre ellos, ni la evolución temporal de las variables incluidas en las estimaciones. Con el objeto de superar estas limitaciones, en este trabajo se analizó la hipótesis de la $\beta$ convergencia en un panel de datos de 23 países de América Latina durante el período 1960-1998. Los resultados obtenidos sugieren la existencia de divergencia absoluta, a la vez que muestran que las economías latinoamericanas no convergen hacia un estado estacionario común (no existe $\beta$ convergencia absoluta). Tampoco se verifica convergencia cuando se incorporan las variables de control del estado estacionario más utilizadas en la literatura (no existe $\beta$ convergencia condicional, sin efectos fijos).

A su vez, tampoco se encuentra evidencia sobre de clubes de convergencia; ninguna de las estimaciones presenta "efectos cruzados" ni variables dummy significativas. De esta manera, las condiciones iniciales de las economías, en lo que respecta a su nivel de producto per cápita, no afectan significativamente a la tasa de crecimiento. En consecuencia, no se verifica la convergencia o divergencia entre grupos de países ni dentro de cada grupo, por lo que la tasa de crecimiento de las economías latinoamericanas sería afectada por factores específicos de cada país. En efecto, en el caso de incluir efectos fijos individuales en las estimaciones no se verifica la existencia de una relación negativa entre el nivel del producto per cápita inicial y la tasa de crecimiento del mismo ( $\beta$ convergencia condicional). En otras palabras, cada país converge a su propio estado estacionario. Este resultado es nuevo con respecto a los estudios empíricos previos sobre convergencia en la región, e indican una discrepancia creciente entre los niveles de ingreso de los países de la región, lo que es compatible con la divergencia entre países encontrada a nivel general.

Una posible extensión de este estudio es la consideración de la posible endogeneidad de las variables explicativas. Por ello una próxima línea de investigación podría ser la utilización del método GMM (estimador del Método General de los Momentos) ya utilizado por Caselli, Esquivel y Lefort (1996). Este estimador tiene la ventaja de solucionar el problema de endogeneidad de las variables explicativas a la vez que considera los efectos fijos específicos de cada país.

Por último, la presencia de efectos fijos significativos sugiere considerar variables de control adicionales. Levine y Renelt (1992) aseguran que más de cin- 
cuenta variables diferentes han resultado significativas en la estimación de la tasa de crecimiento. Algunas de ellas, como por ejemplo indicadores de inestabilidad política, la variación de los términos de intercambio y ciertas medidas de las distorsiones de mercado, pueden tener gran relevancia en el contexto latinoamericano y pueden ser presentadas como alternativas para condicionar el estado estacionario de estas economías. Un estudio empírico que contemple un abanico más amplio de factores socioeconómicos puede permitir una mejor aproximación a la ausencia casi permanente de un proceso de convergencia en este tipo de economías.

\section{REFERENCIAS BIBLIOGRAFICAS}

Artelaris, P, Arvanitidis, P., \& Petrakos, G. (2011). Convergence patterns in the world economy: exploring the nonlinearity hypothesis. Journal of Economic Studies, 38 (3), 236 - 252.

Azomahou, T, El Ouardighi, J., Nguyen-Van, P., \& Cuong Pham, T. (2011). Testing convergence of European regions: A semiparametric approach. Economic Modelling, 28(3), 1202-1210.

Barrientos Quiroga, P. (2010). Convergence Patterns in Latin America. School of Economics and Management, Aarhus University, Economics working paper 2010-15.

Barro, R. (1991). Economic growth in a Cross Section of Countries. Quarterly Journal of Economics, 106(2), 407-443.

Barro, R., (1997). Determinants of economic growth - A cross-country empirical study. Cambridge, Mass: MIT Press.

Barro, R., \& John-Wha Lee (1993). International comparisons of educational attainment. Journal of Monetary Economics, 32, 363-394.

Barro, R., \& John-Wha Lee. (2000). International data on educational attainment: updates and implications, Manuscript, Harvard University.

Barro, R., \& Sala-i-Martin, X. (1992). Convergence. Journal of Political Econo$m y, 100,223-251$.

Baumol, W. (1986). Productivity Growth, Convergence, and Welfare: What the long-run data show. American Economic Review, 76(5), 1072-1085.

Cáceres, L. \& Nuñez Sandoval, O. (1999). Crecimiento económico y divergencia en América Latina. El trimestre económico, volumen LXVI (4), 264.

Cárdenas, M., \& Pontón A. (1995). Growth and convergence in Colombia 19501990. Journal of Development Economics, 47, 5-37.

Caselli, F., Esquivel, G., \& Lefort, F. (1996). Reopening the convergence debate: a new look at cross-country growth empirics. Journal of Economic Growth, 1 (3), 363-390. 
Cavenaile, L., \& Dubois, D. (2011). An empirical analysis of income convergence in the European Union. Applied Economics Letters, Volume 18(17), 1705-1708.

Cellini, R. (1997). Growth empirics: evidence from a panel of annual data. Applied Economic Letters, 4, 347-351.

Cherodian, R., \& Thirlwall, A. (2013). Regional Disparities in Per Capita Income in India: Convergence or Divergence? University of Kent, School of Economics Discussion Papers.

Cuestas, J.C., Monfort, M., \& Ordóñez, J. (2012). Real convergence in Europe: a cluster analysis. Department of Economics, University of Sheffield. Working Paper.

De la Fuente (1998). Algunas técnicas para el análisis de la convergencia con una aplicación a las regiones españolas. Dirección general de análisis y programación presupuestaria. D-98007.

De Long, B. (1988). Productivity growth, convergence and welfare: comment. American Economic Review, 78, 1138-1154.

Dobson, S., \& Ramlogan, C. (2002). Economic growth and convergence in Latin America. Journal of Development Studies, 38, 83-104.

Dobson, S., Goddard, J., \& Ramlogan, C. (2003). Convergence in developing countries: evidence from panel unit root tests. University of Otago, Economic Discussion Papers, 0305.

Duncan, Fuentes (2006). Regional Convergence in Chile: new tests, old results. Cuadernos de Economía (81-112), 2006

Ferreira, A. (1998). Concentraçao regional e dispersao das rendas per capita estaduais; um comentario. Cedepelar, FACE, Universidade Federal de Minas Gerais.

Galor, O. (1996). Convergence? Inferences from theoretical models. Economic Journal, 106, 1056-1069.

Helliwell, J., \& Chung, A. (1992). Convergence and growth linkages between North and South. NBER, Working paper, 3948.

Hu, J. (2011). New Empirical Evidence on Economic Convergence. Journal of Cambridge Studies, 6(4), 103-116.

Islam, N. (1995). Growth empirics: a panel data approach. Quarterly Journal of Economics, 110, 1127-1170.

Levine, R., \& Renelt, D. (1992). A sensitivity analysis of cross-country growth regressions. American Economic Review, 82(4), 942-963.

Lee, K., Pesaran, H., \& Smith, R. (1997). Growth and convergence in multi-country empirical stochastic Solow model. Journal of Applied Econometrics, 12(4), 357-392 
Madariaga, N., Montout, S., \& Ollivaud, P. (2003). Regional convergence, trade liberalization and aglomeration of activities: an analysis of NAFTA and Mercosur cases. Maison de Sciences Economiques, Université Paris.

Mankiw, N., Romer, D., \& Weil, R. (1992). A contribution to the empirics of economic growth. Quarterly Journal of Economics, 107(2), 407-437.

Marina, A., Garrido, N. \& Sotelsek, D. (2000). Dinámica de la distribución del producto a través de las provincias argentinas. 1970-1995. Anales de la XXV Reunión anual de la Asociación Argentina de Economía Política.

Marina, A; Garrido, N. \& Sotelsek, D. (2000). Dinámica de la distribución del producto a través de las provincias argentinas. 1970-1995. Anales de la XXV Reunión anual de la Asociación Argentina de Economía Política.

Quah, D. (1993). Empirical cross-section dynamics in economic growth. European Economic Review, 37, 426-434.

Rabanal, C. (2012). Análisis de la convergencia económica internacional durante el periodo 1950-2009. Revista de Economía Mundial, 31, 167-197.

Rincón Piedrahita, A. (1998). Crecimiento económico en América Latina. Estudio basado en el modelo neoclásico. El trimestre económico, $L X V(3), 259$.

Rodrik, D. (2011). The future of Economic Convergence, NBER Working Paper 17400 .

Rodrik, D. (2011). Unconditional Convergence, NBER Working Paper No. 17546.

Sala-i-Martin, X. (1996). Regional Cohession: evidence and theories of regional growth and convergence. European Economic Review, 40, 1325 - 1352.

Sperlich, S., \& Sperlich, Y. (2012). Growth and Convergence in South-South Integration Areas: Empirical Evidence. Working Paper Series (WPS) 12032.

Utrera, G. \& Koroch, J. A. (1998): Convergencia: evidencia empírica para las provincias argentinas (1953-1994). Anales de la XXIII Reunión Anual de la Asociación Argentina de Economía Política.

Young, A, Higgins, M., \& Levy, D. (2013). Heterogeneous convergence. Economics Letters, 120 (2), 238-241 